\title{
Wave Propagation in Stratified Random Media
}

\author{
Yung Ming Chen
}

\author{
Contribution From the Division of Mathematical Sciences, Purdue University, Lafayette, Ind.
}

(Received December 10, 1963; revised January 6, 1964)

\begin{abstract}
The problem of wave propagation in stratified media is reexamined from the stochastic point of view by introducing the concept of random media. For a small inhomogeneity, this problem is investigated by utilizing the effective refractive coefficient of Chen [1964]. For a large inhomogeneity, this problem is treated by subdividing the medium into many parallel homogeneous layers of random media and utilizing the derived reflection and transmission coefficients of Chen [1964]. In each case, it is found that the small randomness has no drastic and unexpected effects on the behavior of the wave motion in the media considered. Hence, our reliance on the results obtained by a nonrandom approach is increased.
\end{abstract}

\section{Introduction}

The subject of wave propagation through a nonrandom medium has been investigated thoroughly by many authors [Brekhovskikh, 1960; Wait, 1962]. Since, in practice, the properties of the medium are either impossible to be measured accurately or subjected to random variations due to noise, humidity, wind velocity, thermal fluctuations, etc., this problem should be reexamined from the stochastic point of view. Hence, the concept of random medium is introduced.

The wave motion in a transmission medium is described by a vector-valued function $\bar{u}(\bar{r}, t)$ of the position vector $\bar{r}$ and the time $t$. As a consequence of the physical laws governing the wave motion, $\bar{u}(\bar{r}, t)$ satisfies certain partial differential equations of symmetric hyperbolic type. The effect of the transmission medium on the wave motion is characterized by a vector-valued function $\bar{N}(\bar{r}, t)$ the "propagation coefficient." For a random medium the coefficient $\bar{N}(\bar{r}, t, q)$ depends also upon a parameter $g$, ranging over a space $\Omega$ in which a probability density $P(q)$ is defined. If $\bar{u}(\bar{r}, t, q)$ describes the wave motion in a random medium, then the mean value of $\bar{u}(\bar{r}, t, q)$ is defined by $\langle\bar{u}\rangle=\int_{\Omega} P(q) \bar{u}(\bar{r}, t, q) d q$.

In order to distinguish the many phases of random media, we write $\bar{N}(\bar{r}, t, q)=k(\bar{r}, t)$ $\bar{n}(\bar{r}, t, g)$. Then "homogeneous continuous random medium" is defined as $k(\bar{r}, t)$ being a constant. "Inhomogeneous continuous random medium" is defined as $k(\bar{r}, t)$ being a continuous function of $\bar{r}$ and $t$. Finally, "discontinuous random medium" is defined as $k(\bar{r}, t)$ being a discontinuous function of $\bar{r}$ and $t$, and $\bar{n}(\bar{r}, t, q)$ may have different $P(q)$ 's on the two sides of the discontinuity. By the above definitions, we observe that the random stratified media can be either inhomogeneous continuous random media or discontinuous random media.

Many works have been done on the wave propagation in homogeneous continuous random media [Chernov, 1960; Keller, 1960 and 1963; Furutsu, 1963]. However, their results fail to apply to the problem of wave propagation in random stratified media. It has not been until recently in a paper by Y. M. Chen [1964], that the effective refractive coefficient of a slightly inhomogeneous continuous random medium and the reflection and transmission coefficients for the wave propagation in a discontinuous random medium have been obtained; now one can honestly investigate the problem of wave propagation in a random stratified medium.

In this paper we shall study the problem of wave propagation in stratified media of small randomness. For a small inhomogeneity, this problem can be treated by utilizing the effective refractive coefficient of Chen [1964]. The solutions of this type are obtained for two wellknown special profiles of the propagation coefficient [Landau and Lifshitz, 1958]. For a large inhomogeneity, this problem can be effectively treated by subdividing the medium into a num- 
ber of parallel homogeneous layers of random media and utilizing the derived reflection and transmission coefficients of Chen [1964]. In fact, by taking a sufficiently large number of such layers of decreasing thickness, any desired degree of precision may be obtained. In each case, the results are compared with those for the nonrandom media and no significant differences are found. For simplicity, only scalar waves of harmonic time dependence, $e^{-i \omega t}$, are considered.

\section{Reflection and Transmission of Plane Waves From Horizontally Stratified Random Media of Small Inhomogeneity With Special Profiles}

If $r$ denotes a point in three dimensional space, then $u(\bar{r}, q)$, characterizing the motion of plane in a random stratified medium, satisfies the following reduced wave equation

$$
\nabla^{2} u(r, q)+k^{2}[(1+\epsilon f(\bar{r}))(1+\epsilon w(r, q))]^{2} u(r, q)=0
$$

where $\epsilon$ is a small parameter, $f(\bar{r})$ is a continuous function of $\bar{r}$ and $w(\bar{r}, q)$ is a continuous function of $\bar{r}$ and $q$. From Chen [1964], by assuming $\langle w\rangle=0$, it is found that up to and including terms of order $\epsilon^{2}$, the mean value of $u(\bar{r})$ satisfies the following differential equation:

$$
\begin{gathered}
\nabla^{2}\langle u\rangle+\tilde{k}^{2}(1+\epsilon f(\bar{r}))^{2}\langle u\rangle=0, \\
\tilde{k}^{2}=k^{2}\left(1+\epsilon^{2}\left\langle w^{2}\right\rangle K\right),
\end{gathered}
$$

where

$$
K=1-2 i k \int_{0}^{\infty}\left(e^{i 2 k r}-1\right) C(r) d r,
$$

and the correlation coefficient

$$
C\left(\left|\bar{r}-\bar{r}^{\prime}\right|\right)=\frac{\left\langle w(\bar{r}) w\left(\bar{r}^{\prime}\right)\right\rangle}{\left\langle w(\bar{r})^{2}\right\rangle}
$$

is assumed to be a function of the distance $\left|\bar{r}-\bar{r}^{\prime}\right|$ only.

Even for $k$ being real and positive, the imaginary of $\tilde{k}$ can be shown to be positive and its real part can be shown to be greater than $\left(1+\epsilon^{2}\left\langle w^{2}\right\rangle K\right) k$ [Keller, 1963]. Hence the amplitude and phase velocity of the coherent wave, $\langle u\rangle$, is exponentially attenuated and reduced respectively by the randomness of the medium.

Now, $f(\bar{r})$ is assumed to be a function of $y$ only, and $u(\bar{r})=u(y)$ represents a plane wave, coming from $-\infty$, propagating along the $y$-axis. Hence, upon omitting the term $\epsilon^{2} f(y)^{2},(2)$ becomes

$$
\frac{\partial^{2}}{\partial y^{2}}\langle u\rangle+\tilde{k}^{2}(1+2 \epsilon f(y))\langle u\rangle=0 \text {. }
$$

If $(1+2 \epsilon f(y))$ approaches constants $U^{2}$ and $V^{2}$ as $y \rightarrow-\infty$ and $y \rightarrow+\infty$ respectively ( $U^{2}$ may or may not equal $V^{2}$, then

and

$$
\langle u\rangle \sim C e^{i \tilde{k} V y} \text { as } y \rightarrow \infty,
$$

$$
\langle u\rangle \sim A e^{i \tilde{k} U y}+B e^{-i \tilde{k} U y} \text { as } y \rightarrow-\infty .
$$

In this case, the reflection and transmission coefficients are defined as

and

$$
R=A^{-1} B
$$

respectively.

$$
T=C
$$

Case (a) :

$$
(1+2 \epsilon f(y))=1-\frac{\epsilon}{1-e^{-\alpha y}} .
$$



form

In order to find the reflection coefficient, one has to find a solution of (6) which has the

$$
\langle u\rangle=\text { constant } \cdot e^{i \widetilde{K}(1-\epsilon)^{\frac{1}{2} y}} \text { as } y \rightarrow \infty .
$$

By introducing a new variable

and seeking a solution of the form

$$
p=-e^{-\alpha y}
$$

$$
\langle u\rangle=p^{-i \tilde{k}\left(1-\epsilon^{\frac{1}{2}} \alpha-1\right.} \Psi(p)
$$

(where $\Psi(p)$ tends to a constant as $p \rightarrow 0$, i.e., $y \rightarrow \infty$ ), one finds that $\Psi(p)$ satisfies the following: hypergeometric differential equation

$$
p(1-p) \Psi^{\prime \prime}+\left[1-2 i \tilde{k}(1-\epsilon)^{1 / 2} \alpha^{-1}\right](1-p) \Psi^{\prime}-\epsilon \tilde{k}^{2} \alpha^{-2} \Psi=0,
$$

which has as its solution the hypergeometric function

$$
\Psi=\operatorname{MF}\left(i\left[1-(1-\epsilon)^{1 / 2}\right] \tilde{k} \alpha^{-1},-i\left[1+(1-\epsilon)^{1 / 2}\right] \tilde{k} \alpha^{-1},-2 i \tilde{k}(1-\epsilon)^{1 / 2} \alpha^{-1}+1 ; p\right),
$$

where $M$ is an arbitrary constant. This function satisfies the imposed condition, i.e., as $p \rightarrow 0, \Psi \rightarrow 1$. By using the asymptotic form of $\Psi$ as $p \rightarrow-\infty$, one obtains the asymptotic form of $\langle u\rangle$ as $y \rightarrow-\infty$

where

$$
\langle u\rangle \sim(-1)^{-i \tilde{k}(1-\epsilon)^{\frac{1}{2}} \alpha^{-1}}\left[D_{1} e^{i \tilde{k} y}+D_{2} e^{-i \tilde{k} y}\right]
$$

and

$$
D_{1}=\frac{\Gamma\left(-2 i \tilde{k} \alpha^{-1}\right) \Gamma\left(-2 i \tilde{k} \alpha^{-1}(1-\epsilon)^{\frac{1}{2}}+1\right)}{\Gamma\left[-i \tilde{k}\left(1+(1-\epsilon)^{\frac{1}{2}}\right) \alpha^{-1}\right] \Gamma\left[-i \tilde{k}\left(1+(1-\epsilon)^{\frac{1}{2}}\right) \alpha^{-1}+1\right]}
$$

One finally obtains

$$
D_{2}=\frac{\Gamma\left(2 i \tilde{k} \alpha^{-1}\right) \Gamma\left(-2 i \tilde{k} \alpha^{-1}(1-\epsilon)^{\frac{1}{2}}+1\right)}{\Gamma\left[i \tilde{k}\left(1-(1-\epsilon)^{\frac{1}{2}}\right) \alpha^{-1}\right] \Gamma\left[i \tilde{k}\left(1-(1-\epsilon)^{\frac{1}{2}}\right) \alpha^{-1}+1\right]} .
$$

$$
R=\frac{D_{2}}{D_{1}}=\frac{\Gamma\left(2 i \tilde{k} \alpha \alpha^{-1}\right) \Gamma\left[-i \tilde{k}\left(1+(1-\epsilon)^{\frac{1}{2}}\right) \alpha^{-1}\right] \Gamma\left[-i \tilde{k}\left(1+(1-\epsilon)^{\frac{1}{2}}\right) \alpha^{-1}+1\right]}{\Gamma\left(-2 i \tilde{k} \alpha^{-1}\right) \Gamma\left[i \tilde{k}\left(1-(1-\epsilon)^{\frac{1}{2}}\right) \alpha^{-1}\right] \Gamma\left[i \tilde{k}\left(1-(1-\epsilon)^{\frac{1}{2}}\right) \alpha^{-1}+1\right]} .
$$

Since $\operatorname{Im}(k-k)>0$ and $\operatorname{Re} k>\left(1+\epsilon^{2}\left\langle w^{2}\right\rangle K\right) \operatorname{Re} k$ [Keller, 1963], if one keeps away from the poles and zeros of the gamma functions, the above reflection coefficient $R$ is just an analytic continuation of the reflection coefficient for this medium with randomness removed. For all the practical cases, the arguments of the above gamma functions do not equal $\infty, 0,-1,-2$, $-3, \ldots$ Thus, the small randomness of the medium only changes and shifts the amplitude and phase of the reflection coefficient respectively.

Case (b) :

$$
(1+2 \epsilon f(y))=1-\frac{\epsilon}{\cosh ^{2}\left(y \beta^{-1}\right)} .
$$

In order to find the transmission coefficient, one has to find the solution of (6). By making the substitution

where

$$
\langle u\rangle=\left[\cosh \left(y \beta^{-1}\right)\right]^{-2 \lambda} \Psi(p),
$$

and

$$
\lambda=\frac{1}{4}\left[\left(1-4 \beta^{2} \tilde{k}^{2} \epsilon\right)^{1 / 2}-1\right]
$$

$$
p=-\sinh ^{2}\left(y \beta^{-1}\right),
$$

one finds that $\Psi(p)$ satisfies the following hypergeometric differential equation

$$
p(1-p) \Psi^{\prime \prime}+\left[\frac{1}{2}-(1-2 \lambda) p\right] \Psi^{\prime}-\left(\lambda^{2}+\frac{1}{4} \beta^{2} \tilde{k}^{2}\right) \Psi=0 .
$$


Thus

$\langle u\rangle=C_{1}\left[\cosh \left(y \beta^{-1}\right)\right]^{-2 \lambda} F\left[-\lambda+i \frac{1}{2} \tilde{k} \beta,-\lambda-i \frac{1}{2} \tilde{k} \beta, \frac{1}{2} ;-\sinh ^{2}\left(y \beta^{-1}\right)\right]$

$$
+C_{2}\left[\cosh \left(y \beta^{-1}\right)\right]^{-2 \lambda} \sinh \left(y \beta^{-1}\right) F\left[-\lambda+i \tilde{k} \frac{1}{2} \beta+\frac{1}{2},-\lambda-i \frac{1}{2} \tilde{k} \beta+\frac{1}{2}, 1 \frac{1}{2} ;-\sinh ^{2}\left(y \beta^{-1}\right)\right] .
$$

The coefficients $C_{1}$ and $C_{2}$ are determined from the condition that $\langle u\rangle \sim e^{i \tilde{k} y}$ as $y \rightarrow+\infty$. Upon using the proper asymptotic forms of the hypergeometric functions, one obtains

and

$$
\langle u\rangle \sim(-1)^{2 \lambda}\left[\left(C_{1} J_{1}-C_{2} J_{2}\right)\left(-\frac{1}{2}\right)^{-i \tilde{\kappa} \beta} e^{i \tilde{\kappa} y}+\left(C_{1} K_{1}-C_{2} K_{2}\right)\left(-\frac{1}{2}\right)^{i \tilde{\kappa} \beta} e^{-i \tilde{k} y}\right] \text { as } y \rightarrow-\infty,
$$

$$
\langle u\rangle \sim\left[\left(C_{1} J_{1}+C_{2} J_{2}\right)\left(\frac{1}{2}\right)^{-i \tilde{k} \beta} e^{-i \tilde{k} y}+\left(C_{1} K_{1}+C_{2} K_{2}\right)\left(\frac{1}{2}\right)^{i \tilde{k} \beta} e^{i \tilde{k} y}\right] \text { as } y \rightarrow+\infty,
$$

where

$$
\begin{aligned}
J_{1} & =\frac{\pi^{\frac{1}{2}} \Gamma(-i \tilde{k} \beta)}{\Gamma\left(-\lambda-i \frac{1}{2} \tilde{k} \beta\right) \Gamma\left(\lambda+\frac{1}{2}-i \frac{1}{2} \tilde{k} \beta\right)}, \\
J_{2} & =\frac{\pi^{\frac{1}{2}} \Gamma(-i \tilde{k} \beta)}{2 \Gamma\left(-\lambda+\frac{1}{2}-i \frac{1}{2} \tilde{k} \beta\right) \Gamma\left(\lambda+1-i \frac{1}{2} \tilde{k} \beta\right)} \\
K_{1} & =\frac{\pi^{\frac{1}{2}} \Gamma(i \tilde{k} \beta)}{\Gamma\left(-\lambda+i \frac{1}{2} \tilde{k} \beta\right) \Gamma\left(\lambda+\frac{1}{2}+i \frac{1}{2} \tilde{k} \beta\right)},
\end{aligned}
$$

and

$$
K_{2}=\frac{\pi^{\frac{1}{2}} \Gamma(i \tilde{k} \beta)}{2 \Gamma\left(-\lambda+\frac{1}{2}+i \tilde{k} \frac{1}{2} \beta\right) \Gamma\left(\lambda+1+i \frac{1}{2} \tilde{k} \beta\right)} .
$$

The condition that as $y \rightarrow+\infty$ only the transmitted wave is present leads to the following relation between $C_{1}$ and $C_{2}$

Finally,

$$
C_{1} J_{1}+C_{2} J_{2}=0
$$

$$
\begin{aligned}
T & =\frac{\left(C_{1} K_{1}+C_{2} K_{2}\right)(-1)^{-2 \lambda}}{\left(C_{1} J_{1}-C_{2} J_{2}\right)}\left(-\frac{1}{4}\right)^{i \tilde{k} \beta} \\
& =(-1)^{-2 \lambda}\left(-\frac{1}{4}\right)^{i \tilde{k} \beta} \frac{K_{1} J_{2}-J_{1} K_{2}}{2 J_{1} J_{2}} .
\end{aligned}
$$

As in case (a), if one keeps away from the poles and zeros of the gamma functions, the above transmission coefficient $T$ is just an analytic continuation of the transmission coefficient for this medium with the randomness removed. Hence, the small randomness of the medium only changes the amplitude and shifts the phase of the transmission coefficient.

\section{Reflection of a Plane Wave From Horizontally Stratified Random Media of Large Inhomogeneity}

Any stratified random medium of large inhomogeneity can be approximated by a sufficiently large number of parallel homogeneous layers of random media to any degree of accuracy. First, the same problem for the nonrandom media is formulated. For the time being, the number of layers is taken to be $M$ and they lie horizontally below the $x-z$ plane (fig. 1). A plane wave,

$$
u_{i n}=e^{i k_{0} x \sin \theta+i k_{0} y \cos \theta},
$$

is incident at an angle $\theta$ on the upper surface of the first layer. The wave motion in the $m$ th layer below the $x-z$ plane is characterized by the solution of one of the following partial dif- 


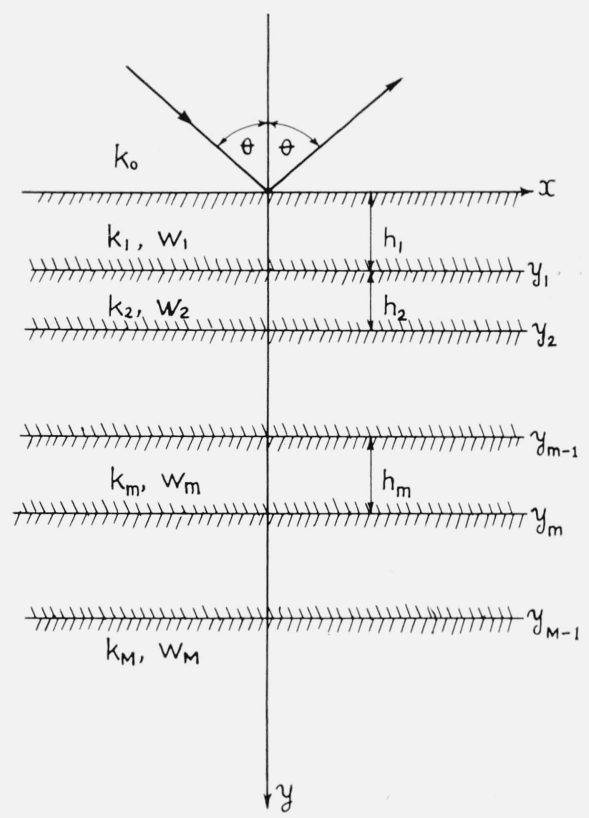

FIGURE 1. The structure of a stratified random medium consists of $\mathrm{M}$ layers of homogeneous random media.

ferential equations,

$$
\left(\nabla^{2}+k_{m}^{2}\right) u_{m}=0, \quad m=1,2,3,4, \ldots, M,
$$

where $k_{m}$ is the propagation constant with nonnegative imaginary part, and the boundary conditions at the interfaces $y=0, y_{1}, y_{2}, y_{3}, \ldots, y_{M-1}$ (fig. 1) are expressed by

$$
\left.\begin{array}{l}
u_{m-1}=u_{m}, \\
\beta_{m-1} \frac{\partial}{\partial y} u_{m-1}=\beta_{m} \frac{\partial}{\partial y} u_{m},
\end{array}\right\} \text { at } y=y_{m-1} \quad m=1,2,3, \ldots, M,
$$

where $\beta_{m}$ is a constant determined solely by the properties of the $m$ th layer.

Because of the randomness in these media, it is more convenient to discuss $\left\langle u_{m}\right\rangle$, the mean of $u_{m}$, than $u_{m}$ itself. From Chen [1964], if one assumes $\left\langle w_{m}\right\rangle=0$ and $k_{m-1} \sim k_{m}, m=1,2,3, \ldots$, $M$ (this implies that the division of medium has to be very fine such that $\left\langle R_{2}\right\rangle$ and $\left\langle T_{2}\right\rangle$ of (85) of Chen [1964] are negligible, one finds that up to and including terms of order $\epsilon^{2}$ (perturbation parameter), away from the boundary surfaces $\left\langle u_{m}\right\rangle$ satisfies (2), (3), (4), and (5) with the subscript $m$ inserted for everything except $\epsilon, \bar{r}$, and $r$, and at the boundary surfaces, $\left\langle u_{m}\right\rangle$ obeys (37).

Now we have the form of the general solution as

$$
\left\langle u_{m}\right\rangle=\left(a_{m} e^{\tilde{i s_{m}}}+b_{m} e^{-i \tilde{s}_{m}^{y}}\right) e^{i \tilde{\lambda} x}, \quad m=1,2,3, \ldots, M,
$$

where $\tilde{k}_{m}^{2}=\tilde{s}_{m}^{2}+\tilde{\lambda}_{2}$, and $\tilde{\lambda}$ can take any value. Upon imposing the outgoing wave condition on (38) for $m=M$ and inserting (35) into (38) for $m=0$, it follows that $b_{M}=0$ and $a_{0}=1, \tilde{s}_{0}=$ $k_{0} \cos \theta, \tilde{\lambda}=k_{0} \sin \theta$. Upon applying the boundary conditions (37) to (38), the coefficient $b_{0}$ is obtained as

$$
b_{0}=\frac{-i k_{0} \beta_{0} \cos \theta-z_{1}}{-i k_{0} \beta_{0} \cos \theta+z_{1}}
$$


where

$$
\left.\begin{array}{l}
z_{1}=s_{1} \beta_{1} \frac{z_{2}-s_{1} \beta_{1} \tan \tilde{s}_{1} h_{1},}{s_{1} \beta_{1}+z_{2} \tan \tilde{s}_{1} h_{1}} \\
z_{2}=s_{2} \beta_{2} \frac{z_{3}-s_{2} \beta_{2} \tan \tilde{s}_{2} h_{2},}{s_{2} \beta_{2}+z_{3} \tan \tilde{s}_{2} h_{2}} \\
\ldots \ldots \\
z_{m}=s_{m} \beta_{m} \frac{z_{m+1}-s_{m} \beta_{m} \tan \tilde{s}_{m} h_{m}}{s_{m} \beta_{m}+z_{m+1} \tan \tilde{s}_{m} h_{m}}, \\
\ldots \\
z_{M-1}=-i s_{M-1} \beta_{M-1} \frac{s_{M} \beta_{M}+s_{M-1} \beta_{M-1} \tan \tilde{s}_{M-1} h_{M-1}}{s_{M-1} \beta_{M-1}+s_{M} \beta_{M} \tan \tilde{s}_{M-1} h_{M-1}}
\end{array}\right\}
$$

and $h_{m}$ is the thickness of the $m$ th layer.

Since $\left|\tilde{s}_{m}-s_{m}\right|$ is very small, if one keeps away from the singularities of tan $\left(\tilde{s}_{m} h_{m}\right)$ and of the denominator of $z_{m}$, one would expect no drastic change in $b_{0}$. It is also interesting to notice that in this case $b_{0}$ is not an analytic continuation of the reflection coefficient for this medium with randomness removed as in section 2 .

\section{References}

Brekhovskikh, L. M. (1960), Waves in layered media, translated from the Russian by David Lieberman, translation ed., Robert T. Beyer, p. 561 (Academic Press, New York, N.Y.).

Chen, Y. M. (1964), Wave propagation in inhomogeneous and discontinuous random media, J. Math. Phys. (in press).

Chernov, L. A. (1960), Wave propagation in a random medium, translated from the Russian by R. A. Silverman, p. 168 (McGraw-Hill Book Co., Inc., New York, N.Y.).

Furutsu, K. (May-June 1963), On the statistical theory of electromagnetic waves in a fluctuating medium. I, J. Res. NBS 6\%D (Radio Prop.), No. 3, 303-323.

Keller, J. B. (1960), Wave propagation in random media, Proceedings of the 13th Symposium on Applied Math., pp. 227-246 (American Math. Society, New York, N.Y.).

Keller, J. B. (1963), Stochastic equations and wave propagation in random media, Proceedings of the 14th Symposium on Applied Math. (American Math. Society, New York, N.Y.).

Landau, L. D. and E. M. Lifshitz (1958), Quantum mechanics, nonrelativistic theory pp. $72-77$ (AddisonWesley Publishing Co., Inc., Reading, Mass.).

Wait, J. R. (1962), Electromagnetic waves in stratified media (Macmillan Co., New York, N.Y.).

(Paper 68D6-372) 